\title{
Labyrinthe
}

18 | 2004 (2)

La Recherche dans tous ses éclats

\section{« Esthétique du témoignage »}

ACI Réseau des MSH Caen, Poitiers, Toulouse-le-Mirail, université de Caen, 18-20 mars 2004

\section{Grégoire Leménager}

\section{(2) OpenEdition}

12 Journals

Édition électronique

URL : http://journals.openedition.org/labyrinthe/221

DOI : $10.4000 /$ labyrinthe.221

ISSN : 1950-6031

Éditeur

Hermann

Édition imprimée

Date de publication : 15 juillet 2004

Pagination : 75-78

Référence électronique

Grégoire Leménager, « "Esthétique du témoignage » », Labyrinthe [En ligne], 18| 2004 (2), mis en ligne le 24 juin 2008, consulté le 30 avril 2019. URL : http://journals.openedition.org/labyrinthe/221 ; DOI : 10.4000/labyrinthe.221 


\title{
«ESTHÉTIQUE DU TÉMOIGNAGE»
}

\section{ACI Réseau des MSH Caen, Poitiers, Toulouse-le-Mirail, université de Caen, 18-20 mars 2004}

\author{
Grégoire LEMÉNAGER \\ g.lemenager@oreka.com
}

D'un chaos l'autre, le $\mathrm{xx}^{\mathrm{e}}$ siècle a littéralement dynamité les formes de représentation héritées du passé. Brutalement devenues inadéquates, mensongères ou indécentes devant des cataclysmes historiques qui les dépassent, elles ont laissé place à l'émergence massive, dans l'urgence, de ce que l'on peut appeler, faute de mieux, le genre du témoignage. Faute de mieux, car le concept générique reste fuyant devant la variété des formes qu'emprunte le témoignage - diversité des supports, dispositifs et types de discours, mais aussi des stratégies déployées par le témoin pour convaincre de sa bonne foi, partager une expérience et, souvent, transfigurer le document en un monument dédié aux victimes de l'événement. Le paradoxe semble en effet constitutif de la notion: si la volonté de témoigner se définit a priori par une relation d'immédiateté aux faits, dans une quête d'authenticité et de transparence, cette volonté ne doit pas moins composer avec l'inévitable opération de mise en forme que requiert, de manière plus ou moins délibérée, tout processus de communication pour toucher son but.

Ce paradoxe d'une «esthétique du témoignage» était au cœur des enjeux du colloque organisé sous ce titre par Carole Dornier à l'université de Caen. Bel exemple de travail collectif, cette rencontre essentiellement consacrée au $\mathrm{XX}^{\mathrm{e}}$ siècle s'inscrivait dans un programme de recherches plus vaste, mis sur pied autour de la notion de témoignage par des universitaires de Caen, Poitiers et Toulouse ${ }^{1}$; bien que dominé par une approche littéraire, le colloque associait des chercheurs venus

1. Pour plus de précisions, voir : http://www.unicaen.fr/mrsh/identites/temoignage/index.php. Cette rencontre était la troisième d'une série dont la quatrième devrait avoir lieu les 2 et 3 décembre 2004 à Poitiers, sous l'intitulé : «Crédibilité et vraisemblance du témoignage». 
de la linguistique, de l'histoire, de la sociologie, des études théâtrales et cinématographiques. Chacun, à sa façon, s'est confronté aux problèmes que la mise en forme du témoignage semble soulever: problèmes d'ordre éthique, épistémique et esthétique, répertoriés par Marie-Louise Ollé (Toulouse) ${ }^{2}$.

Problème éthique, d'abord: dès lors qu'il s'empare d'une matière tragique ou grave, le témoignage paraît moralement incompatible avec l'idée de recherche esthétique, qui, par définition, suppose la création d'un objet destiné à séduire par sa beauté. Au nom de quoi, et comment s'autoriser à «récupérer» la douleur des autres, de ceux qui souvent sont morts, à des fins artistiques toujours suspectes de quémander une gloire personnelle ? L'intervention de Carine Trévisan (Paris VII) a bien montré l'importance du problème en examinant le pudique «art de l'écoute» mis en œuvre par Henri Calet en 1945, pour recueillir dans Les Murs de Fresnes les graffitis laissés dans leurs geôles par les déportés. En analysant le regard porté par le girondin Louis-Sébastien Mercier sur les dérives de la Révolution française, Yolande Coste (Poitiers) a suggéré que le problème ne se posait peut-être pas alors de façon si aiguë: l'usage de procédés rhétoriques tirait encore sa légitimité d'une subordination de la nécessité de toucher à la volonté d'instruire. En creux, cette excursion hors du $\mathrm{XX}^{\mathrm{e}}$ siècle désignait comme l'une de ses spécificités, née de la Première Guerre mondiale, le malaise moral suscité par l'esthétisation du témoignage. L'attention portée par plusieurs intervenants - et notamment par Renaud Dulong (EHESS) à l'exigeante conception du témoignage développée par Jean-Norton Cru dans les années 1920 allait incontestablement dans ce sens. En effet, le sentiment de scandale proclamé par celui-ci stigmatisait en premier lieu les best-sellers de l'époque qui, au lieu de faire l'apologie du pacifisme, bâtissaient leur succès en métamorphosant la boue sanglante des tranchées en une vision héroïque de la guerre.

Mais dans le prolongement de cette réflexion éthique, la normatisation des récits de guerre compilés par Cru soulève la question de la valeur épistémique du témoignage. L'historien américain Leonard V. Smith (Oberlin College) s'est ainsi penché sur «l'esthétique de l'objec-

2. C'est à propos de témoignages sur la guerre civile du Guatemala que Marie-Louise Ollé a emprunté cette tripartition à Greimas. 
tivité» réclamée par Cru qui, statistiques à l'appui, s'attachait à dénoncer la falsification du réel opérée par les représentations sublimées de l'enfer des tranchées. Le témoignage, dans un sens strict qui rejoint celui de sa définition juridique, ne peut être outil de connaissance que s'il se tient dans les limites de la fidélité aux faits et au vécu. Or, Ruth Amossy (Tel-Aviv) s'est, de son côté, interrogée sur les applications de tels préceptes, en observant que les textes d'infirmières de guerre, bruts et indifférents à tout travail de recomposition des faits, ne faisaient souvent que reconduire à leur insu la figure préconçue de l'infirmière dévouée, proche de l'image d'Épinal répandue alors par la CroixRouge... La transparence et l'objectivité sont évidemment des vœux pieux, ne serait-ce que parce que tout témoignage vise à persuader; ce qu'ont remarquablement dégagé Luc Vigier (Poitiers), à propos de textes d'Aragon dédiés aux martyrs de la Résistance, et Michèle Bokobza-Kahan (Tel-Aviv), au sujet de témoignages rassemblés par les avocats des convulsionnaires, au XVIII ${ }^{\mathrm{e}}$ siècle. Tous deux se sont intéressés aux effets produits par la mise en recueil - au rôle de la présentation, des redondances, de la signature.

Surtout, le langage a ses pièges que le témoin ne repère pas toujours, et qui peuvent saper la possibilité même de sa démarche. Françoise Dubor (Poitiers) l'a rappelé en s'appuyant sur le procès d'une «opacité » de la parole instruit par Marguerite Duras dans L'Amante anglaise. Comme le disait Perec, «les faits ne parlent pas d'eux-mêmes ». En ce sens, l'élaboration esthétique, loin d'être une ornementation incompatible avec la transmission de la vérité, serait au contraire une condition sine qua non de sa réalisation. Jorge Semprun ne disait pas autre chose à propos de l'expérience concentrationnaire : «Seul l'artifice d'un récit maîtrisé parviendra à transmettre partiellement la vérité du témoignage.» D'où la nécessité d'inventer un langage, comme dans le cas particulier de l'œuvre mescalinienne de Henri Michaux, présenté par Anne Gourio (Caen). D'où, comme l'a mis au jour Philippe Mesnard (Marne-la-Vallée) chez Gradowski et Kertész, le recours à «l'articulation des points de vue» pour dire l'expérience des camps dans ce qu'elle a de déstructurant pour la perception et le jugement. D'où l'intérêt, aux yeux mêmes des sociologues selon Guillaume Grandazzi et Frédéric Lemarchand (Caen), de «l'artialisation du témoignage» pour appréhender une catastrophe aussi confuse et diffuse que celle de Tchernobyl. 
Ainsi la fiction, congédiée par une réalité qui la dépasse, resurgirait-elle au sein de tout témoignage. Mais ce constat permet-il de balayer l'hypothèse d'un genre du témoignage fondé sur l'identité narrateur/auteur et le récit scrupuleusement factuel? François Rastier (Paris VII) s'est engagé dans cette voie polémique en présentant la poésie de Primo Levi: la distinction factuel/fictionnel ne serait pas opératoire à ses yeux pour appréhender la notion de témoignage - présupposé qui a également guidé Jennifer Kilgore (Caen) dans sa lecture à la loupe d'un poème de Geoffrey Hill évoquant la déportation. Laure Himy (Caen) a poussé le paradoxe en s'appuyant sur Perec et Bober pour montrer que, la remémoration se fondant sur une rupture à soi-même, le témoignage sur le passé ne serait pas soluble dans l'imaginaire, mais devrait au contraire emprunter ses voies. Une idée stimulante, mais acrobatique: rejoignant la plupart des participants, le cinéaste Emmanuel Finkiel (venu présenter son beau film Voyages) a vigoureusement plaidé pour que l'on évite de confondre fiction et documentaire. Du moins ces interventions à contre-courant signalentelles combien sont floues les frontières entre témoignage et œuvre d'art: Denise Schropfer (Paris X) l'a confirmé, en décrivant le dispositif théâtral de Rwanda 94, qui intégrait sur scène la présence d'une rescapée du génocide; Anthony Fiant (Caen) s'est interrogé sur le rôle de la subjectivité de l'auteur dans les documentaires récents de Claude Lanzmann, Rithy Panh, Chantal Akerman et Frederick Wiseman; l'auscultation du cas Hervé Guibert a permis à Bruno Blanckeman de distinguer un régime «ordinaire» du témoignage, et un régime «littéraire» fondé sur une surdétermination de ses différentes composantes.

Enfin, la majorité des communications a souligné l'importance de la réception du témoignage, seule capable de statuer sur sa valeur esthétique, mais surtout seule à pouvoir le valider comme tel. FrançoisCharles Gaudard a relu sous cet angle les faux témoignages sur Waterloo légués par Chateaubriand, Stendhal et Hugo. Il a ainsi ouvert la voie à Dominique Moncond'huy (Poitiers) et Michaël Rinn (Brest) qui, à propos d'évocations des camps allant d'Antelme à Kertész, ont particulièrement insisté sur la responsabilité éthique du lecteur luimême. Dépositaire du témoignage, c'est à lui de devenir, au présent, témoin à son tour. 